\title{
Collisionless shocks in laser-produced plasma generate monoenergetic high-energy proton beams
}

\author{
Dan Haberberger ${ }^{1}$, Sergei Tochitsky' ${ }^{1}$ Frederico Fiuza ${ }^{2}$, Chao Gong ${ }^{1}$, Ricardo A. Fonseca ${ }^{2,3}$, \\ Luis O. Silva ${ }^{2}$, Warren B. Mori ${ }^{1}$ and Chan Joshi ${ }^{1 \star}$
}

Compact and affordable ion accelerators based on laser-produced plasmas have potential applications in many fields of science and medicine. However, the requirement of producing focusable, narrow-energy-spread, energetic beams has proved to be challenging. Here we demonstrate that laser-driven collisionless shocks can accelerate proton beams to $\sim 20 \mathrm{MeV}$ with extremely narrow energy spreads of about $1 \%$ and low emittances. This is achieved using a linearly polarized train of multiterawatt $\mathrm{CO}_{2}$ laser pulses interacting with a gas-jet target. Computer simulations show that laser-heated electrons launch a collisionless shock that overtakes and reflects the protons in the slowly expanding hydrogen plasma, resulting in a narrow energy spectrum. Simulations predict the production of $\sim 200 \mathrm{MeV}$ protons needed for radiotherapy by using current laser technology. These results open a way for developing a compact and versatile, high-repetition-rate ion source for medical and other applications.

aser-accelerated proton sources are under active study because of their potential application to radiotherapy of cancerous tumours ${ }^{1,2}$, radiography ${ }^{3}$, generation of short-lived isotopes needed in positron emission tomography ${ }^{4}$, injectors for conventional accelerators ${ }^{5}$, and inertial confinement fusion ${ }^{6}$. The goal of this research is to produce a compact and affordable source of focusable, high-energy proton beams. In addition, certain applications such as hadron therapy and injection into conventional accelerators require a narrow-energy-spread proton beam. In this paper, we demonstrate through experiments and simulations a new scheme for generating high-quality monoenergetic proton beams for these applications: acceleration by collisionless shocks ${ }^{7-9}$ in laser-produced hydrogen plasmas. The study of acceleration by shock waves is also important in astrophysical and space plasmas ${ }^{10,11}$.

At present, the two most studied mechanisms for laser-driven ion acceleration are target-normal sheath acceleration (TNSA; refs $12-14)$ and radiation-pressure acceleration ${ }^{15,16}$. These are known to produce a large number of energetic ions in a very short distance ${ }^{17}$. The relatively well understood TNSA process has produced characteristic exponential energy spectra with a highest proton energy of $\sim 60 \mathrm{MeV}$ (ref. 18). Recently two groups have had limited success in obtaining a peak in the TNSA-generated spectrum with a full-width at half-maximum (FWHM) energy spread $\Delta E / E_{\mathrm{FWHM}} \sim 20 \%$ by engineering the target geometry or composition, but the ion energies were limited to a few $\mathrm{MeV}$ (refs 19,20). Spectral selection through external focusing ${ }^{21,22}$ as well as radiofrequency phase rotation ${ }^{23}$ has also been applied to generate a narrow peak from a TNSA exponential spectrum.

The radiation-pressure acceleration mechanism has the potential to generate narrow-energy-spread ion beams, but the data are scant, with the only result reported so far yielding $\mathrm{C}^{6+}$ ions with an energy of $\sim 30 \mathrm{MeV}$ and a $\Delta E / E_{\mathrm{FWHM}} \sim 50 \%$ (ref. 24 ). The majority of the recent work on laser-driven ion acceleration a
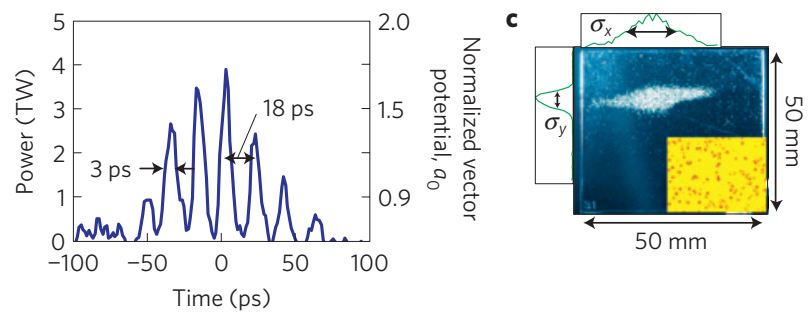

b

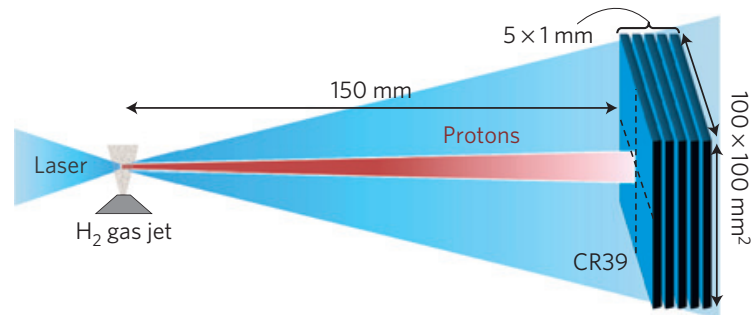

Figure 1 | Experimental setup, the $\mathrm{CO}_{2}$ laser pulse profile and an image of a CR39 detector. a, The temporal profile of the $\mathrm{CO}_{2}$ laser pulse as measured by the streak camera (see Supplementary Discussion). The micropulses have an FWHM of 3 ps and a separation of 18 ps. $\mathbf{b}$, Schematic representation of the experimental arrangement for proton detection. $\mathbf{c}$, An image of the proton beam on the fourth CR39 detector ( $22 \mathrm{MeV}$ ). The inset shows a magnified portion of the CR39, indicating no significant overlap of individual proton tracks. $\sigma_{x}=5.7 \mathrm{~mm}$ and $\sigma_{y}=2.2 \mathrm{~mm}$ are the FWHM dimensions of the proton beam in the $x$ and $y$ directions respectively.

has been carried out with either $0.8-\mu \mathrm{m}$ - or $1-\mu \mathrm{m}$-wavelength laser pulses less than one picosecond long, interacting with foil targets with one exception. In the latter, a circularly polarized, 8 ps $\mathrm{CO}_{2}$ laser pulse interacting with a gaseous target produced $1 \mathrm{MeV}$ protons with a $\Delta E / E_{\mathrm{FWHM}} \sim 10 \%($ refs 25,26$)$.

\footnotetext{
${ }^{1}$ University of California Los Angeles, UCLA Engineering Receiving, 420 Westwood Plaza, Los Angeles, California 90095, USA, ${ }^{2}$ GoLP/Instituto de Plasmas e Fusao Nuclear-LaboratorioAssociado, Instituto Superior Técnico, 1049-001 Lisbon, Portugal, ${ }^{3}$ Instituto Universitario de Lisboa (ISCTE-IUL), 1649 -026 Lisbon, Portugal. *e-mail: cjoshi@ucla.edu.
} 


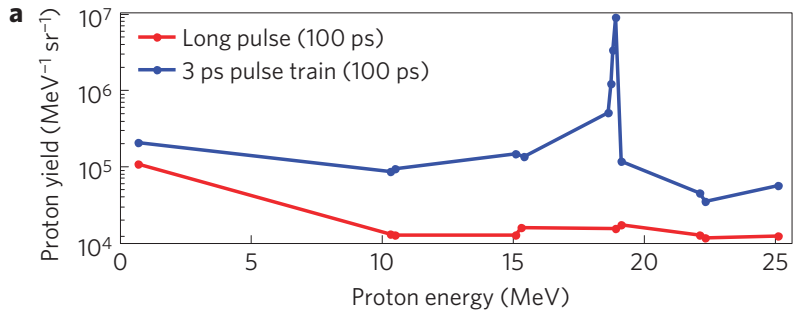

b

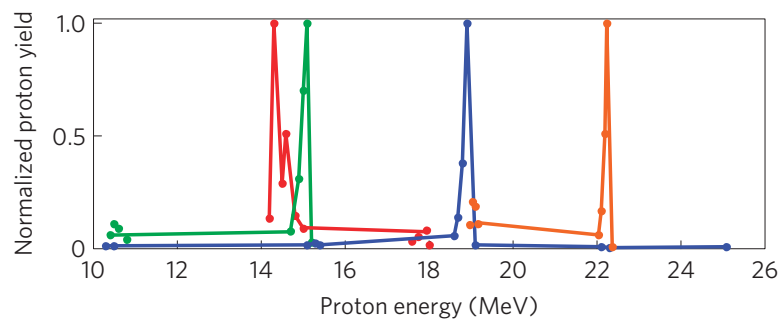

Figure 2 | Proton energy spectra. a, Proton spectra obtained with a 100-ps-long laser pulse (red) and a 100 ps macropulse consisting of a number of 3 ps micropulses (blue) both containing $60 \mathrm{~J}$. The typical noise level on a single CR39 detector was 100 pits. The total number of protons contained within the monoenergetic peak was $2.5 \times 10^{5}$. b. The details of the energy spectra on four different laser shots with different macropulse structures (number of pulses and $a_{0}$ values ranging from 1.5 to 2.5 )

In this paper we show the generation of $\sim 20 \mathrm{MeV}$ proton beams with a $\Delta E / E_{\mathrm{FWHM}}<1 \%$ using an entirely different mechanism: collisionless shock-wave acceleration in a hydrogen plasma. The laser pulse from the Neptune $\mathrm{CO}_{2}$ laser system at the University of California at Los Angeles ${ }^{27,28}$, containing up to $60 \mathrm{~J}$, is focused using a $40^{\circ}$ off-axis parabolic mirror onto a nominally $1-\mathrm{mm}$-diameter supersonic hydrogen gas jet to a spot size radius of $w_{0}=60 \mu \mathrm{m}$. The $\sim 100$ ps envelope of the laser macropulse is modulated with a series of micropulses, each $\sim 3$ ps long and separated by $18 \mathrm{ps}$ as shown in Fig. 1a. This modulation of the laser macropulse occurs naturally owing to the periodic gain modulation of the $\mathrm{CO}_{2}$ molecule ${ }^{27}$. In addition, a single smooth $100 \mathrm{ps}$ laser pulse can be obtained by simply seeding a 100 -ps-long $10 \mu \mathrm{m}$ pulse into the amplification stages instead of a 3-ps-long $10 \mu \mathrm{m}$ pulse. The peak normalized vector potential $a_{0}$ is typically $\sim 1.5-2.5$ (intensity, $I<6.5 \times 10^{16} \mathrm{~W} \mathrm{~cm}^{-2}$ ) for the two largest laser micropulses. The temporal evolution of the plasma density profile is monitored by on-line interferometry using a $2 \mathrm{ps}, 532 \mathrm{~nm}$ laser pulse. The relative timing between the $\mathrm{CO}_{2}$ macropulse and the interferometry pulse, as well as the structure of the micropulses, was monitored with a streak camera (see Supplementary Information).

The forward-propagating ions are recorded by a stack of five $1-\mathrm{mm}$-thick CR39 detectors placed $15 \mathrm{~cm}$ from the plasma, as shown in Fig. 1b. After each laser shot, the detectors are etched in $\mathrm{NaOH}$ solution, then the ion number is counted and pit diameters are measured using automated microscope-based apparatus. As described in the Methods section, by analysing the penetration depth of the protons in the CR39 plastic, we are able to recover their spectrum.

In Fig. 1c we show an image of the expanding proton beam seen on the fourth $1 \mathrm{~mm}$ CR39 detector with a nominal energy of $22 \mathrm{MeV}$. This beam is contained within a solid angle that is an order of magnitude smaller than that subtended by the laser beam. The transverse spatial profile of the proton beam seems to be correlated to that of the incident laser beam (at the point of reflection from the plasma as described below). A measurement of the $\mathrm{CO}_{2}$ laser beam profile in vacuum for this focusing condition uncovered a strong astigmatism of the laser beam, which is then apparently transferred to the accelerated

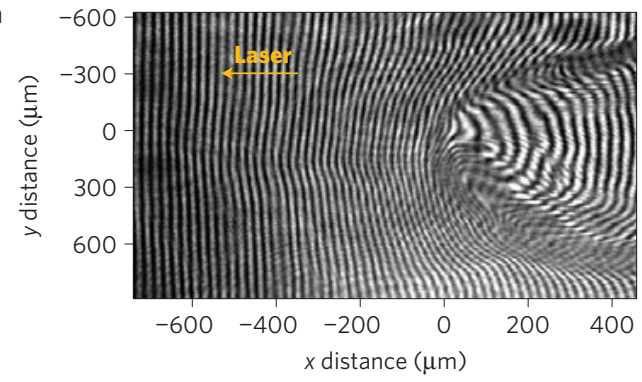

b

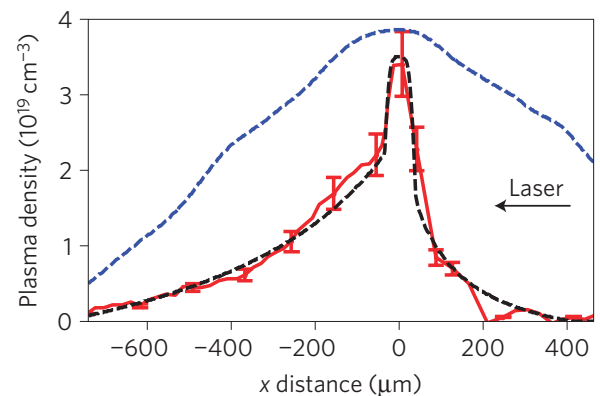

Figure 3 | Laser-produced plasma profile. a, Interferogram of the plasma taken at the peak of the $\mathrm{CO}_{2}$ laser macropulse showing the hole bored into the plasma by the laser pulse. b. The initial gas-jet density profile (dashed blue), the plasma density profile extracted from the interferogram in a (solid red) and the simulated plasma density profile (dashed black). The experimental error in the extracted plasma density profile is estimated by the addition of a possible unseen fringe near the peak of the profile where the interferogram is slightly distorted.

proton beam. From the size of the beam in the two transverse directions and assuming an initial source size equal to the laser spot size $(60 \mu \mathrm{m})$, we have estimated the geometric emittances (which together with the energy spread determine the ultimate focusability) of this beam to be $\varepsilon_{x}=\theta_{x} w_{0}=1.1 \mathrm{~mm} \mathrm{mrad}$ by $\varepsilon_{y}=0.4 \mathrm{~mm}$ mrad, where $\theta_{x, y}$ is the half-angle divergence of the proton beam in the $x$ or $y$ direction. This corresponds to normalized emittances of $\varepsilon_{\mathrm{n}, x}=\beta \gamma \varepsilon_{x}=250 \mu \mathrm{mmrad}$ by $\varepsilon_{\mathrm{n}, y}=90 \mu \mathrm{m} \mathrm{mrad}$, where $\beta=v / c$ is the proton velocity normalized to the speed of light and $\gamma$ is the relativistic Lorentz factor. By scanning the focal position, more symmetric (albeit larger) emittance proton beams were obtained. The spectra of these beams are shown in Fig. 2. The typical geometric emittances of these beams are $5 \mathrm{~mm}$ mrad.

The complete spectrum of the protons obtained for a smooth $100 \mathrm{ps}$ laser pulse $\left(a_{0} \sim 0.3\right)$ and a microstructured laser pulse $\left(a_{0} \sim 2\right)$ containing the same energy are plotted in Fig. 2 a. We can clearly see that for the smooth pulse the ion spectrum is monotonically decreasing, whereas for the structured pulse there is a well-defined peak with a less-than-1\% (FWHM) energy spread. This energy spread corresponds to an $\sim 10 \mathrm{ps}$ pulse duration at the CR39 detector, which is much shorter than those produced by conventional accelerators. An example of four proton spectra all showing a narrow energy spread but energies varying between 15 and $22 \mathrm{MeV}$ is presented in Fig. $2 \mathrm{~b}$. The spectra are obtained for the same laser energy but different macropulse widths, resulting in different numbers of micropulses and the peak $a_{0}$ varying in the range of 1.5-2.5. Therefore, the proton energy variation is due to a combination of these two factors. For the optimal experimental conditions (peak plasma density, $n_{\mathrm{e}}$, is three to five times the critical plasma density for $10 \mu \mathrm{m}$ light, $n_{\mathrm{cr}}$, and the laser is focused on the initial position of the $n_{\mathrm{cr}}$ layer), the observed spectra had a monoenergetic feature even though the proton energy varied with the shotto-shot fluctuations of the laser. These results show a marked 
a
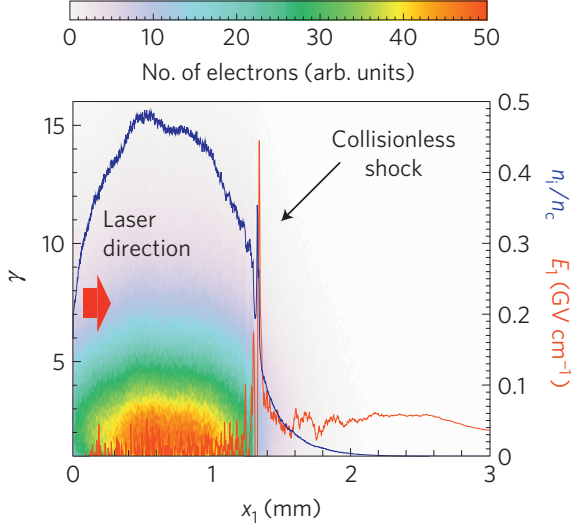

C

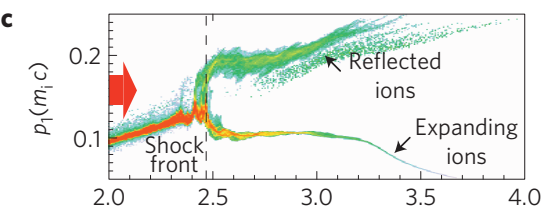

d

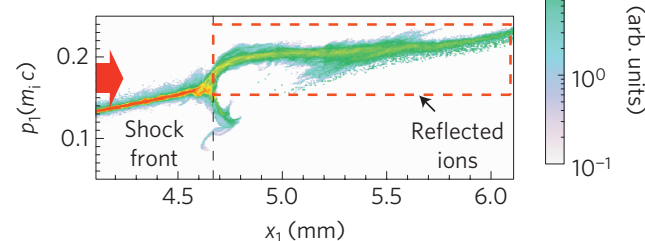

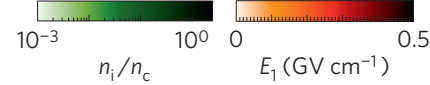
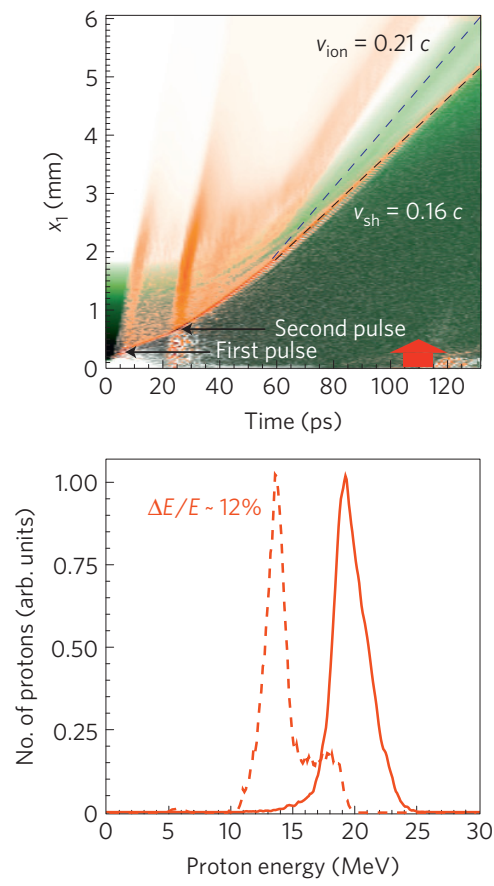

Figure 4 | Simulation results. a, Plasma-electron phase-space (colour bar), transversely averaged ion-density (blue) and longitudinal electric-field (red) profiles after 46 ps. The electric-field spike is due to the shock and the smaller plateau field ahead of the shock is from TNSA. $\mathbf{b}$, Time evolution of the ion density (green) and longitudinal electric field $\left(E_{1}\right)$ (orange). The two strong features at 7 and 25 ps are from the electrons heated by the two laser pulses.

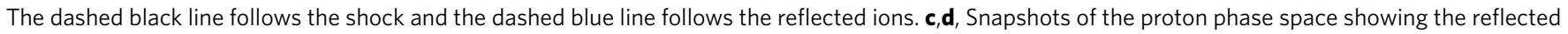
ions inside the expanding plasma at $73 \mathrm{ps}$ (c) and outside the expanding plasma at 120 ps (d). e, Spectrum of the reflected proton bunch for an $a_{0}$ of 2.5 (red solid line) taken from the red dotted box in $\mathbf{d}$ after $120 \mathrm{ps}$ and for an $a_{0}$ of 2 (red dashed line).

improvement in the peak energy (at a comparable laser $a_{0}$ ) and an order-of-magnitude improvement in the energy spread of proton beams obtained in both TNSA and radiation-pressure acceleration experiments.

The temporal evolution of the plasma has been tracked using laser interferometry. Figure $3 a$ shows an interferogram of the plasma density profile taken at the peak of the $\mathrm{CO}_{2}$ laser macropulse with the extracted on-axis plasma density plotted in Fig. $3 \mathrm{~b}$ (solid red). It is found that the early micropulses serve to ionize the gas jet as early as 80 ps before the peak of the macropulse by tunnel ionization and the plasma density profile is strongly steepened on the front side of the target by the radiation pressure of the laser ${ }^{29,30}$. The plasma electrons during the early micropulses, $a_{0} \ll 1$, are expected to be cold, therefore electron-neutral collisions serve to ionize the rear of the target to form a millimetre-scale-length plasma. As this plasma expands, the plasma density on the rear of the target drops rapidly to generate an exponentially falling density profile. The successful proton acceleration shots showed such a steepened plasma density profile at the peak of the laser macropulse with an overdense $\left(n_{\mathrm{e}}>10^{19} \mathrm{~cm}^{-3}\right)$ peak plasma density at the front and an exponentially decaying density at the back. Confirmation of the extracted plasma density profile (solid red) was obtained by creating a synthetic interferogram (dashed black) to match that in Fig. 3a (see Supplementary Discussion).

The hole-boring effect due to the radiation pressure of the laser micropulses can lead to the acceleration of ions ${ }^{25}$. Using snapshots of the plasma density profile taken at different times but on shots with identical parameters, we determined the average hole-boring velocity (velocity at which the overdense plasma layer is pushed forward by the laser) to be $v_{\mathrm{hb}}=3.2 \pm 0.8 \times 10^{8} \mathrm{~cm} \mathrm{~s}^{-1}$.
An upper estimate of the peak hole-boring velocity calculated using the expression given in ref. 31 is $6.3 \times 10^{8} \mathrm{~cm} \mathrm{~s}^{-1}$ using the experimentally measured peak plasma density of $3 \times 10^{19} \mathrm{~cm}^{-3}$ and assuming a constant laser $a_{0}$ of 2.3. Cold plasma protons that reflect off this moving layer would gain a maximum energy of $(1 / 2) m_{\mathrm{i}}\left(2 v_{\mathrm{hb}}\right)^{2}=213 \mathrm{keV}$ (experiment) or $1 \mathrm{MeV}$ (theory), where $m_{\mathrm{i}}$ is the mass of the proton. These values are more than an order of magnitude smaller than the proton energies observed in the experiment, thus pointing to a different mechanism for proton acceleration.

The mechanism of ion acceleration is elucidated using the particle-in-cell code OSIRIS (ref. 32), which uses a cold preformed plasma with a density profile similar to the measured plasma density profile shown in Fig. 3b. Two linearly polarized 3-ps-long micropulses with an $a_{0}$ of 2.5 and separation of 18 ps are incident on this plasma (see Supplementary Movie). The first laser pulse is absorbed near the critical density, heating up the plasma locally and causing its expansion, which lowers the peak density. As the second pulse arrives, the peak density is of the order of the critical density and the plasma electrons are efficiently heated up to a temperature of $\sim 1.1 \mathrm{MeV}$ in the region leading up to the critical density. The plasma electron temperature is consistent with ponderomotive scaling for the hot electrons, $T_{\text {hot }}=m_{\mathrm{e}} c^{2} \sqrt{1+a_{0}^{2} / 2}=1.04 \mathrm{MeV}$, where $m_{\mathrm{e}}$ is the mass of the electron and $c$ is the speed of light. These electrons launch a collisionless electrostatic shock wave (electron-ion collisional mean free path, $\lambda_{\mathrm{e}-\mathrm{i}}>$ shock thickness $\sim 5 \lambda_{\mathrm{D}}$, where the Debye length $\left.\lambda_{\mathrm{D}}=\left[k T_{\text {hot }} / 4 \pi n_{\mathrm{e}} e^{2}\right]^{1 / 2}\right)$ at the overdense layer that propagates through the plasma (Fig. 4a). The most energetic electrons heated by the first and second laser pulses can be seen to be leaving the plasma at $7 \mathrm{ps}$ and $25 \mathrm{ps}$, 


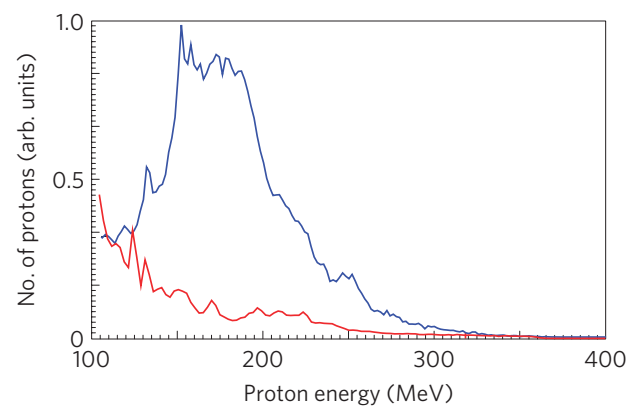

Figure 5 | Simulated proton spectra. Proton spectra obtained in simulations for a single picosecond-long pulse with an $a_{0}$ of 10 (blue curve) and a single 100-ps-long laser pulse with an $a_{0}$ of 2.5 (red curve). The plasma density profile used is similar to that shown in Fig. $3 \mathrm{~b}$ with a peak plasma density of $7 n_{\mathrm{cr}}$ (blue curve) and $3 n_{\mathrm{cr}}$ (red curve).

respectively, through their effect on the longitudinal electric field plotted in Fig. $4 \mathrm{~b}$. The shock propagates through the plasma at a near-constant velocity of $v_{\mathrm{sh}}=0.16 \mathrm{c}$ for almost $100 \mathrm{ps}$ after the arrival of the second laser pulse (Fig. $4 \mathrm{~b}$ ). The shock Mach number, measured in the upstream frame $\left(v_{\text {up }}\right)$ at $73 \mathrm{ps}$ (see Fig. 4c), is given by $M=\left[\left(v_{\mathrm{sh}}-v_{\mathrm{up}}\right) / c_{\mathrm{s}}\right]=\left[\left(v_{\mathrm{sh}}-v_{\mathrm{up}}\right) / c\right] \sqrt{m_{\mathrm{i}} / m_{\mathrm{e}}}\left(1+a_{0}^{2} / 2\right)^{-1 / 4} \sim$ 2 , where $c_{\mathrm{s}}$ is the ion sound speed and using the observed upstream velocity of $0.1 \mathrm{c}$. In this simulation, and indeed in the experiment, the shock is enhanced by the electrons heated by the subsequent laser pulse(s).

As the shock structure overtakes the plasma ions, they are reflected off it as shown in Fig. 4c, and gain a velocity corresponding to twice the shock velocity minus their expansion velocity $(\sim 0.21 c)$. This process forms an ion beam that retains its narrow energy spread as it exits the plasma (Fig. 4d). Note that the sheath field is much reduced at the edge of the expanding long-scale-length plasma, thus minimizing the contribution of the TNSA mechanism to the ion energy ${ }^{33}$. This simulation resulted in the acceleration of protons with a peak energy of $19 \mathrm{MeV}$ and a $\Delta E / E_{\mathrm{FWHM}} \sim 12 \%$ propagating outside the plasma after $100 \mathrm{ps}$ (see Fig. 4e). When the $a_{0}$ was reduced to 2 , the peak proton energy dropped to $15 \mathrm{MeV}$ (also shown in Fig. 4e). This variation of energy is consistent with the variation observed in the experiment (Fig. 2b).

Further simulations using a single 100-ps-long, linearly polarized laser pulse with an $a_{0}=0.5$ (similar to the experimental parameters of Fig. 2a) gave a monotonically decreasing proton spectrum with a maximum energy of $\sim 20 \mathrm{MeV}$ (not shown here). Increasing the peak $a_{0}$ of this laser pulse to 2.5 still produced an exponentially decaying proton spectrum, though with a cut-off energy of $340 \mathrm{MeV}$ as shown in Fig. 5 (red curve). From a large number of simulations we have determined that laser pulses on a few-picosecond timescale are optimal for local heating of the plasma electrons and launching a shock. A train of pulses can apparently do this very efficiently, with the early pulses helping to ionize the gas target and shaping the plasma so that the most intense pulse or pulses heat the electrons and form a shock as explained above.

To evaluate the potential of shock-wave acceleration in gases for reaching very high proton energies, we modelled a large- $a_{0}$ case. For a laser pulse with an $a_{0}$ of $10\left(I=10^{18} \mathrm{~W} \mathrm{~cm}^{-2}\right.$ for a $\mathrm{CO}_{2}$ laser $)$, and an increased peak plasma density of $7 \times 10^{19} \mathrm{~cm}^{-3}$ to compensate for the relativistic transparency due to the higher laser intensity, the proton peak energy increased to $\sim 170 \mathrm{MeV}$ (blue line in Fig. 5), nearing the requirement for proton radiotherapy. Shock-wave acceleration can also be applied for the production of higher-mass ions such as nitrogen and oxygen. Current experimental yields will need to be increased by two to three orders of magnitude for meeting the requirements of hadron therapy and injectors for conventional accelerators even if high-repetition-rate lasers are used. It should be noted that $1-10 \mathrm{~Hz}$ repetition rate lasers with an $a_{0}$ of at least 10 are technologically feasible. Furthermore, $\mathrm{CO}_{2}$ lasers today are capable of generating tens of joules of energy at a kilohertz repetition rate.

\section{Methods}

Proton detection. After each laser shot, the CR39 detectors are etched in fresh $6 \mathrm{~N} \mathrm{NaOH}$ solution at $80^{\circ} \mathrm{C}$ for $5 \mathrm{~h}$. The ion number is then counted and the pit diameters are measured using automated microscope-based apparatus yielding a single data point on either side of the CR39. Monoenergetic features were then identified by a gross change in yield between adjacent data points. In these cases, further etching of the corresponding CR39 in $5 \mathrm{~h}$ increments up to a total of $20 \mathrm{~h}$ was carried out to obtain a finer spectral resolution around the feature.

The energy of a detected proton is calculated by equating the distance travelled through the CR39 plastic to the distance travelled by a proton of a certain energy to yield the Bragg peak at that location ${ }^{34}$. The bulk CR39 plastic etching rate was measured to be $\sim 10 \mu \mathrm{m} \mathrm{h}^{-1}$; therefore, each $5 \mathrm{~h}$ step in etching moves the plane of detection $50 \mu \mathrm{m}$ deeper into the CR39 plastic, corresponding to an incremental change in energy. In addition, we measured a linear increase in pit diameter as a function of time. This enabled us to identify the protons that had a Bragg peak in each $50 \mu \mathrm{m}$ slice of CR39. It should be noted that, at each time step, only new pits with diameters less than $40 \mu \mathrm{m}$ are counted, as larger diameters represent augmented pits from the previous time step. This procedure has a counting error of 200 pits per detector plate. This detection system provides a spectral resolution of $0.1 \mathrm{MeV}$ centred around $20 \mathrm{MeV}$.

Plasma density profile characterization. A supersonic cylindrical gas jet similar to one described in ref. 35 was used for the target. The plasma density profile of the gas target (blue dotted line in Fig. 3b) was measured by using $50 \mathrm{fs}, 0.8 \mu \mathrm{m}$ laser interferometry. The $\mathrm{CO}_{2}$ laser-plasma was probed with a Mach-Zehnder interferometer using a $2 \mathrm{ps}, 532 \mathrm{~nm}$ pulse synchronized with the $\mathrm{CO}_{2}$ macropulse. Synchronization is accomplished through a cross-correlation method using a silicon semiconductor switching technique ${ }^{36}$

The interferogram shown in Fig. 3a contains areas where probe light refracted outside the collection angle has skewed the interference pattern, and therefore necessitated manual analysis to extract the plasma density profile along the laser axis assuming the plasma density profile to be axisymmetric. The comparison of the fringe positions in Fig. 3 a with those of a vacuum interferogram yields the total phase shift along the laser axis. On the back side of the profile $(x<0)$, the plasma density is calculated directly assuming a Gaussian transverse profile and measuring its $1 / e$ characteristic length. On the front side of the profile $(x>0)$, however, the probe light accrues phase from passing through two high-density walls formed by the ponderomotive push of the laser, $\Delta \varphi_{\mathrm{w}}$, and from the low-density cavity left in between, $\Delta \varphi_{\mathrm{c}}$. Therefore, the total phase shift measured on axis can be approximated as $\Delta \varphi_{\mathrm{t}}=\Delta \varphi_{\mathrm{c}}+2 \Delta \varphi_{\mathrm{w}}$. For each position along the laser axis, $\Delta \varphi_{\mathrm{w}}$ is estimated by comparing the fringe shift in the wall with the vacuum interferogram, then subtracted from $\Delta \varphi_{\mathrm{t}}$ to isolate the phase shift due only to the plasma inside the cavity, $\Delta \varphi_{\mathrm{c}}$. Finally, the density on axis is calculated assuming a flat plasma profile inside the cavity. Application of this method to the interferogram in Fig. $3 \mathrm{a}$ results in the density profile shown by the red line in Fig. 3b. Further support for the extracted plasma profile is gained through comparison to a simulated interferogram as presented in Supplementary Discussion.

OSIRIS simulations. Two-dimensional simulations were carried out using the fully relativistic particle-in-cell code OSIRIS 2.0 (ref 32). OSIRIS has been widely used to simulate various ion and electron acceleration scenarios in plasmas and directly compared with experimental results ${ }^{37-39}$. The simulations used a preformed electron-proton plasma with a density profile similar to that measured experimentally and shown in Fig. $3 \mathrm{~b}$ (red solid) at the peak of the $\mathrm{CO}_{2}$ macropulse. It has a linear $0.1 \mathrm{~mm}$ rise and $1 \mathrm{~mm}$ exponential fall, with a peak density of $2.8 \times 10^{19} \mathrm{~cm}^{-3}$. A first set of simulations was carried out using a sequence of two 3 ps $\mathrm{CO}_{2}$ laser pulses with a Gaussian temporal profile and peak $a_{0}=2.5$, separated by $18 \mathrm{ps}$. The simulation results are illustrated by the Supplementary Movie. A second set of simulations used a single $\mathrm{CO}_{2}$ laser pulse with $100 \mathrm{ps}$ pulse width and $a_{0}=0.5$. Finally, a third set of simulations was carried out with an increased laser intensity $\left(a_{0}=10\right)$ and increased peak plasma density of $7 \times 10^{19} \mathrm{~cm}^{-3}$. All simulations used a box size of $6.1 \times 0.8 \mathrm{~mm}^{2}, 12,288 \times 1,536$ cells, cubic interpolation (third-order splines) and nine computational particles per cell per species, totalling $3 \times 10^{8}$ particles. The particles were pushed for $>10^{5}$ iterations.

Received 17 June 2011; accepted 27 September 2011; published online 13 November 2011

\section{References}

1. Bulanov, S. V., Esirkepov, T. Zh., Khoroshkov, V. S., Kuznetsov, A. V. \& Pegoraro, F. Oncological hadrontherapy with laser ion accelerators. Phys. Lett. A 299, 240-247 (2002). 
2. Linz, U. \& Alonso, J. What will it take for laser driven proton accelerators to be applied to tumor therapy? Phys. Rev. STAB 10, 094801 (2007).

3. Borghesi, M. et al. Electric field detection in laser-plasma interaction experiments via the proton imaging technique. Phys. Plasmas 9, 2214-2220 (2002).

4. Spencer, I. et al. Laser generation of proton beams for the production of short-lived positron emitting isotopes. Nucl. Instrum. Methods B-183, 449-458 (2001).

5. Krushelnick, K. et al. Ultrahigh-intensity laser-produced plasmas as a compact heavy ion injection source. IEEE Trans. Plasma Sci. 28, 1110-1115 (2000).

6. Roth, M. et al. Fast ignition by intense laser-accelerated proton beams. Phys. Rev. Lett. 86, 436-439 (2001).

7. Denavit, J. Absorption of high-intensity subpicosecond lasers on solid density targets. Phys. Rev. Lett. 69, 3052-3055 (1992).

8. Zhidkov, A., Uesaka, M., Sasaki, A. \& Daido, H. Ion acceleration in a solitary wave by an intense picosecond laser pulse. Phys. Rev. Lett. 89, 215002 (2002).

9. Silva, L. O., Marti, M., Davies, J. R. \& Fonseca, R. A. Proton shock acceleration in laser-plasma interactions. Phys. Rev. Lett. 92, 015002 (2004).

10. Sagdeev, R. Z. \& Kennel, C. F. Collisionless shock waves. Sci. Am. 264, 106-113 (1991).

11. Adriani, O. et al. PAMELA measurements of cosmic-ray, proton, and helium spectra. (PAMELA collaboration). Science 332, 69-72 (2011).

12. Fuchs, J. et al. Laser-driven proton scaling laws and new paths towards energy increase. Nature Phys. 2, 48-54 (2006).

13. Robson, L. et al. Scaling of proton acceleration driven by petawatt-laser-plasma interactions. Nature Phys. 3, 58-62 (2007).

14. Cowan, T. E. et al. Ultralow emittance, multi-MeV proton beams from a laser virtual-cathode plasma accelerator. Phys. Rev. Lett. 92, 204801 (2004).

15. Esirkepov, T., Borghesi, M., Bulanov, S. V., Mourou, G. \& Tajima, T. Highly efficient relativistic-ion generation in the laser-piston regime. Phys. Rev. Lett. 92, 175003 (2004).

16. Macchi, A., Cattani, F., Liseykina, T. V. \& Cornolti, F. Laser acceleration of ion bunches at the front surface of overdense plasmas. Phys. Rev. Lett. 94, 165003 (2005).

17. Ledingham, K. W. D. \& Galster, W. Laser driven particle and photon beams and some applications. New J. Phys. 12, 045005 (2010).

18. Snavely, R. A. et al. Intense high-energy proton beams from petawatt-laser irradiation of solids. Phys. Rev. Lett. 85, 2945-2948 (2000).

19. Hegelich, B. M. et al. Laser acceleration of quasi-monoenergetic $\mathrm{MeV}$ ion beams. Nature 439, 441-444 (2006).

20. Schwoerer, H. et al. Laser-plasma acceleration of quasi-monoenergetic protons from microstructured targets. Nature 439, 445-448 (2006).

21. Toncian, T. et al. Ultrafast laser-driven microlens to focus and energy-select mega-electron volt protons. Science 312, 410-413 (2011).

22. Schollmeier, M. et al. Controlled transport and focusing of laser-accelerated protons with miniature magnetic devices. Phys. Rev. Lett. 101, 055004 (2008)

23. Noda, A. et al. Phase rotation scheme of laser-produced ions for reduction of the energy spread. Laser Phys. 16, 647-653 (2006).

24. Henig, A. et al. Radiation-pressure acceleration of ion beams driven by circularly polarized laser pulses. Phys. Rev. Lett. 103, 245003 (2009).

25. Palmer, C. A. J. et al. Monoenergetic proton beams accelerated by a radiation pressure driven shock. Phys. Rev. Lett. 106, 014801 (2011).
26. Najmudin, Z. et al. Observation of impurity free monoenergetic proton beams from the interaction of a $\mathrm{CO}_{2}$ laser with a gaseous target. Phys. Plasmas 18, 056705 (2011).

27. Haberberger, D., Tochitsky, S. \& Joshi, C. Fifteen terawatt picosecond $\mathrm{CO}_{2}$ laser system. Opt. Express 18, 17865-17875 (2010).

28. Tochitsky, S. Ya. et al. Efficient shortening of self-chirped picosecond pulses in a high-power $\mathrm{CO}_{2}$ amplifier. Opt. Lett. 26, 813-815 (2001).

29. Fedosejevs, R., Tomov, I. V., Burnett, N. H., Enright, G. D. \& Richardson, M. C. Self-steepening of the density profile of a $\mathrm{CO}_{2}$-laser-produced plasma. Phys. Rev. Lett. 39, 932-935 (1977).

30. Young, P. E. et al. Time-dependent channel formation in a laser-produced plasma. Phys. Rev. Lett. 75, 1082-1085 (1995).

31. Wilks, S. C. \& Kruer, W. L. Absorption of ultrashort, ultra-intense laser light by solids and overdense plasmas. IEEE J. Quantum Electron. 33, 1954-1968 (1997).

32. Fonseca, R. A. et al. OSIRIS, a three-dimensional fully relativistic particle in cell code for modeling plasma based accelerators. Lect. Note Comput. Sci. 2331, 342-351 (2002).

33. Wilks, S. C. et al. Energetic proton generation in ultra-intense laser-solid interactions. Phys. Plasmas 8, 542-549 (2001).

34. Leferve, H. W., Sealock, R. M. \& Connolly, R. C. Response of CR-39 to 2-MeV microbeams of H, He, and Ne. Rev. Sci. Instrum. 53, 1221-1227 (1982).

35. Semushin, S. \& Malka, V. High density gas jet nozzle design for laser target production. Rev. Sci. Instrum. 72, 2961-2965 (2001).

36. Alcock, A. J. \& Corkum, P. B. Ultra-fast switching of infrared radiation by laser-produced carriers in semiconductors. Can. J. Phys. 57, 1280-1290 (1979).

37. Blue, B. E. et al. Plasma-wakefield acceleration of an intense positron beam. Phys. Rev. Lett. 90, 214801 (2003).

38. Tsung, F. S. et al. Simulation of monoenergetic electron generation via laser wakefield accelerators for 5-25 TW lasers. Phys. Plasmas 13, 056708 (2006).

39. Willingale, L. et al. Collimated multi-MeV ion beams from high-intensity laser interactions with underdense plasma. Phys. Rev. Lett. 96, 245002 (2006).

\section{Acknowledgements}

Work supported by DOE Grant DE-FG02-92-ER40727, NSF grant PHY-0936266 at UCLA, European Research Council ERC-2010-AdG Grant 267841 and FCT (Portugal) grants PTDC/FIS/111720/2009 and SFRH/BD/38952/2007. We thank A. Pak, N. Lemos and K. A. Marsh for characterizing the gas-jet targets. Computing resources provided by PRACE (Tier 0) on Jugene based in Germany, the Hoffman Cluster (UCLA) and the IST Cluster (IST Lisbon).

\section{Author contributions}

D.H., S.T., C.G. and C.J. conceived and designed the experiments, carried out the experiments, analysed the data, contributed analysis tools and wrote the paper. F.F., L.O.S., R.A.F. and W.B.M. carried out the numerical simulations and wrote the paper.

\section{Additional information}

The authors declare no competing financial interests. Supplementary information accompanies this paper on www.nature.com/naturephysics. Reprints and permissions information is available online at http://www.nature.com/reprints. Correspondence and requests for materials should be addressed to C.J. 